\title{
Negative Pressure Dressings over Free Muscle Flaps with Immediate Split-Thickness Skin Grafting: A 9-Year Experience
}

\author{
Pallavi A. Kumbla, MD ${ }^{1}$ Steven L. Henry, MD ${ }^{2}$ Carter J. Boyd, BS $^{3}$ Patrick K. Kelley, MD 4
} Ashley Q. Thorburn, MD ${ }^{5}$ René P. Myers, MD

${ }^{1}$ UAB Division of Plastic Surgery, Birmingham, Alabama

${ }^{2}$ Seton Institute of Reconstructive Plastic Surgery, Austin, Texas

${ }^{3}$ UAB School of Medicine, Birmingham, Alabama

${ }^{4}$ Craniofacial Team of Texas, Austin, Texas

${ }^{5}$ David Grant Medical Center, Travis AFB, California

${ }^{6}$ Division of Pediatric Plastic Surgery, UAB Division of Plastic Surgery,

Birmingham, Alabama

J Reconstr Microsurg Open 2020;5:e27-e31.

\begin{abstract}
Address for correspondence René P. Myers, MD, Division of Pediatric Plastic Surgery, UAB Division of Plastic Surgery, 1600 7th Avenue, South Suite 322, Birmingham, AL 35233 (e-mail: rmyers@uabmc.edu).
\end{abstract}

\begin{abstract}
Keywords

- negative pressure dressing

- free muscle flap

- split-thickness skin graft

Background Negative pressure wound therapy has allowed for significant advances in the treatment of wounds. This occurs through a process of angiogenesis, microdeformation, macrodeformation, and decrease in exudate and bacterial load. It is routinely used as a bolster in the management of skin grafts. However, its role as a dressing over free muscle flaps has not gained widespread acceptance due to the fear of flap compromise.

Methods A retrospective review of 97 patients over 9 years was performed. All patients underwent free muscle flap coverage of various wounds with immediate splitthickness skin grafting. A negative pressure dressing was applied, with windows made in the foam sponge to enable Doppler monitoring as well as visual inspection of the flap. Complications including flap failure, skin graft loss, hematoma, distal flap necrosis, negative pressure dressing failure, partial muscle necrosis, and mild flap congestion were assessed.

Results Flap loss occurred at a rate of $8.2 \%$ (eight flaps). Four of these flaps were lost due to patient factors not attributable to the dressing. In the remaining four flaps ( $4.1 \%$ of the series), it is conceivable that the negative pressure dressing was a contributing factor. However, this failure rate is comparable to flap loss rates in studies where negative pressure dressings were not used. The rates of skin graft failure, hematoma, distal flap necrosis, inability to maintain seal, partial muscle necrosis, and mild flap congestion were also acceptable and similar to studies where negative pressure dressings were not used.

Conclusion Negative pressure dressings over free muscle flaps with immediate splitthickness skin grafts are effective and safe to use while allowing for postoperative flap monitoring and skin graft protection.
\end{abstract}

received

February 1, 2020

accepted after revision

March 20, 2020
DOI https://doi.org/

10.1055/s-0040-1710345. ISSN 2377-0813.
Copyright $\odot 2020$ by Thieme Medical Publishers, Inc., 333 Seventh Avenue, New York, NY 10001, USA. Tel: +1(212) 760-0888.
License terms

(c) (i) 
Since its introduction in the 1990s, negative pressure wound therapy (NPWT) has revolutionized the management and treatment of several different types of wounds. NPWT works in several ways. First, it stimulates angiogenesis around the wound bed. Second, the foam that is placed onto the wound bed facilitates a process known as microdeformation where the wound bed surface takes the same shape as the foam that it is in contact with. Third, macrodeformation occurs in which compressive force reduces edema and stimulates wound contracture, thereby decreasing surface area. Lastly, negative pressure therapy controls exudate and decreases bacterial load. ${ }^{1}$

Negative pressure therapy has been used as a bolster over skin grafts with great success dating back to the 1990s. The success of a skin graft in the immediate postoperative period is dependent on close contact to the wound bed. This may be accomplished with a bolster or large bulky pressure dressing. However, Nakayama et al in 1990 showed that negative pressure therapy was a satisfactory alternative to these traditional methods. Initially, they applied a thin layer of silicone gauze to the bed of the skin graft, followed by suction drains and a drape. They were able to achieve excellent coaptation of the graft to the wound bed as well as remove fluid from the wound bed that may have been a potential source of graft failure. ${ }^{2}$ Blackburn et al in 1998 also confirmed excellent results with use of a negative pressure dressing with greater than $95 \%$ graft take in all patients in their study. ${ }^{3}$ Only recently has negative pressure therapy over free tissue transfer gained momentum due to fear of impacting anastomotic patency from the compressive force exerted by negative pressure therapy. Chim et al performed a prospective study from 2014 to 2016 evaluating the use of immediate negative pressure therapy in free muscle coverage of lower extremity trauma. In their study, they found that edema and flap thickness was reduced in the immediate postoperative period after a negative pressure dressing was placed at $75 \mathrm{~mm} \mathrm{Hg}$ continuously compared with patients who had wet-to-dry dressings. This difference was statistically significant, and they reported only one flap loss in the group treated with negative pressure therapy. However, this loss was unrelated to the type of dressing and was rather from an undiagnosed deep vein thrombosis. ${ }^{4}$ Despite the small sample size of their study and the lack of long-term follow-up, they were able to demonstrate that negative pressure dressings placed over free muscle transfer can be a safe dressing that not only decreases edema but also does not compromise anastomotic patency. In another study by Bi et al, a retrospective review was performed over a 3-year period to evaluate the use of negative pressure dressings following skin-containing free tissue transfers. In their study, 24 patients underwent free tissue transfer. In total, 11 of the 24 patients were lower extremity reconstructions. There were no complications related to the use of negative pressure therapy and no flap losses, which once again demonstrates that use of negative pressure immediately postoperatively does not increase flap loss or compromise the pedicle supplying the flap. ${ }^{5}$ Eisenhardt and associates examined 26 patients with NPWT lower extremity soft tissue defects observing just two flap failures both occurring in patients with comorbid peripheral vascular disease. ${ }^{6}$ In our retrospective, multi-institutional study performed over 9 years, we were able to review 97 cases of negative pressure therapy utilized immediately over split-thickness skin grafted free muscle flaps. This is the largest series to date, and we are able to show not only that negative pressure therapy following free muscle transfer safe, but also that it does not impede monitoring and that it allows for excellent skin graft survival.

\section{Methods}

A retrospective review of 97 patients was performed over a 9-year period. Patients were accrued from three different surgeons at two different hospital systems and ranged from ages 4 to 84 years old. Inclusion criteria encompassed all patients receiving a free muscle flap from various donor sites including gracilis, latissimus dorsi, rectus abdominis, soleus, and vastus lateralis with concomitant skin grafting for coverage. Exclusion criteria were if we did not anticipate an excellent seal with negative pressure dressing therapy. Recipient locations included ankle, leg, foot, heel, scalp, back, wrist, forearm, elbow, hand, and arm. A split-thickness skin graft was performed and was sutured or stapled over the free muscle flap. A doppler probe was used to identify arterial signals over the flap, and these were marked with 5-0 prolene sutures. A nonadherent barrier was placed between the skin graft and the negative pressure dressing foam sponge. The sponge was tailored to the general shape of the flap but slightly wider in all dimensions to avoid tension on the flap during activation of the negative pressure. Windows were cut out of the sponge at the sites of the colored sutures to allow for doppler monitoring through the dressing. The sponge was stapled in place around the edges and then the standard clear adhesive tape applied over the sponge. We were very careful to apply the sponge and tape without tension on the flap, especially if the flap surface was convex. Negative pressure was applied from 75 to $125 \mathrm{~mm}$ $\mathrm{Hg}$ continuously (varying by surgeon preference), but the senior author now most commonly utilizes $125 \mathrm{~mm} \mathrm{Hg}$. When indicated, splints and external fixators were utilized in the settings of fractures or for immobilization of a limb with a new flap. The negative pressure dressing was removed on postoperative day 4 or 5 , and the flap and skin graft dressed daily according to the surgeon's personal protocol (-Fig. 1A-E). Free flap monitoring involved hourly Doppler checks and clinical exam for 24 to 48 hours. This was then followed by 24 to 48 hours of Doppler checks every 2 hours. Subsequently, flaps were monitored every 4 hours until discharge. The number of flaps from specific donor sites was documented ( - Table $\mathbf{1}$ ) as well as the number of flaps per recipient location ( $\mathbf{- T a b l e ~} \mathbf{2}$ ). Primary endpoints were flap survival and survival of the skin graft. Complications including flap failure, skin graft failure, hematoma, distal flap necrosis, negative pressure dressing failure, partial muscle necrosis, and mild flap congestion were evaluated. The number of flaps with each complication was recorded, and the percent of flaps in each category was calculated (-Table 3). 


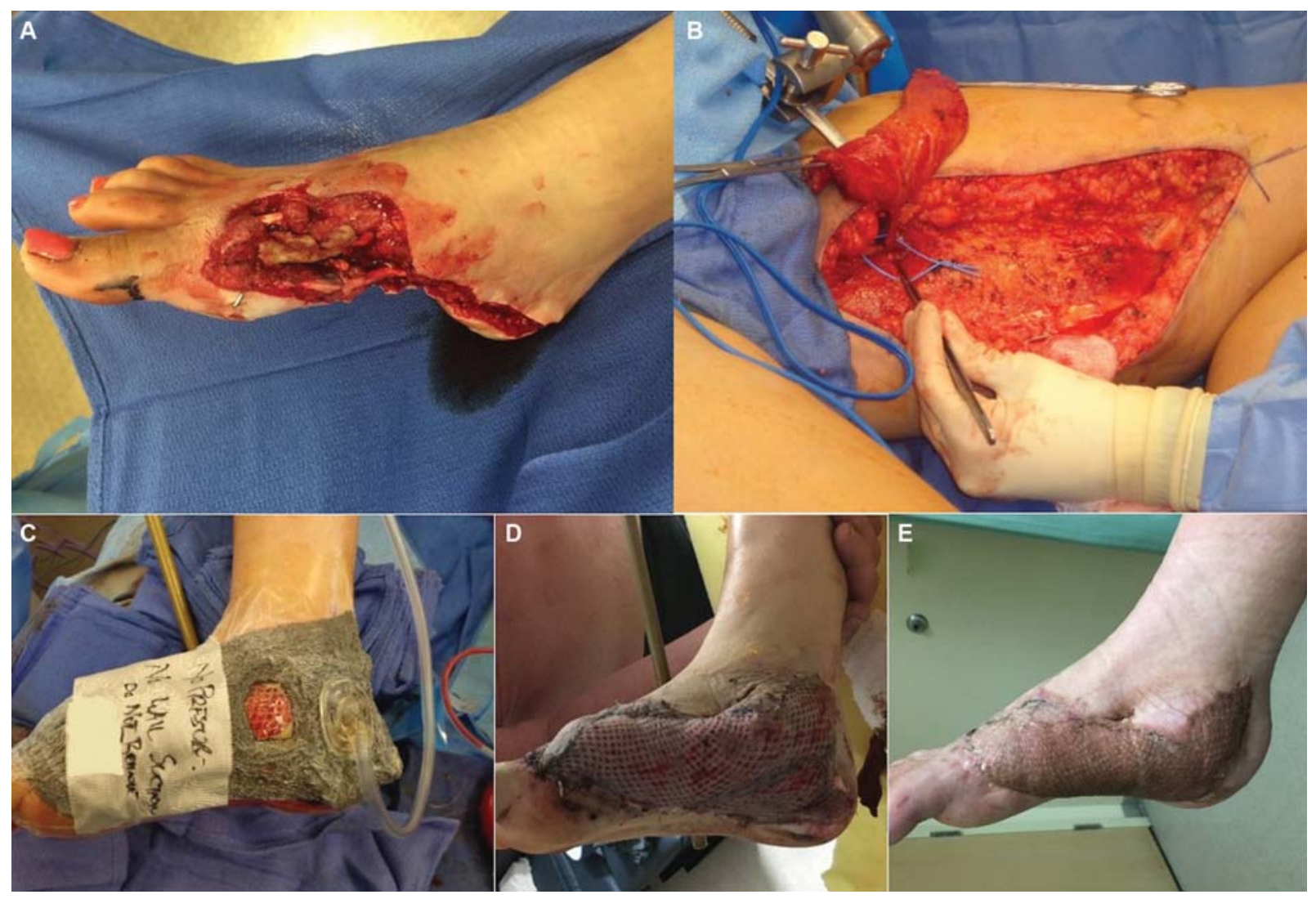

Fig. 1 (A) Lower extremity foot defect requiring reconstruction. (B) Free muscle flap harvest for foot wound reconstruction. (C) Negative pressure dressing with window over skin-grafted muscle flap that allows for Doppler monitoring of flap and visual inspection of flap. (D) Negative pressure dressing removed on postoperative day 4 or 5 with no skin graft or muscle flap compromise. (E) Long-term follow-up demonstrating well-healed skin-grafted free muscle flap.

Table 1 Donor sites used for reconstruction

\begin{tabular}{|l|l|}
\hline Donor Site & Number of flaps \\
\hline Gracilis & 53 \\
\hline Latissimus & 34 \\
\hline Rectus abdominis & 6 \\
\hline Vastus lateralis & 3 \\
\hline Soleus & 1 \\
\hline
\end{tabular}

\section{Results}

Over a 9-year period, 97 free muscle flaps were covered postoperatively with a negative pressure dressing. The vast majority of the donor sites from this series were gracilis muscle or latissimus dorsi muscle (53 and 34, respectively). A minority of the flaps were taken from the rectus abdominis, vastus lateralis, and soleus muscles $(6,3$, and 1 , respectively; - Table 1). The majority of recipient sites were in the lower extremity (-Table 2). Total 16 flaps suffered a complication (-Table 3). Split-thickness skin graft failure occurred in three flaps (3.2\%). Hematoma was found in one flap (1.1\%), as was distal flap necrosis (1.1\%), inability to maintain negative pressure dressing seal (1.1\%), partial muscle flap necrosis (1.1\%), and mild flap congestion (1.1\%). The most serious complication, flap failure, occurred in eight flaps (8.4\%). Of
Table 2 Recipient sites for free muscle coverage

\begin{tabular}{|l|l|}
\hline Recipient Location & Number of flaps \\
\hline Ankle & 37 \\
\hline Leg & 33 \\
\hline Foot & 10 \\
\hline Scalp & 4 \\
\hline Heel & 3 \\
\hline Forearm & 3 \\
\hline Wrist & 2 \\
\hline Back & 2 \\
\hline Hand & 1 \\
\hline Arm & 1 \\
\hline Elbow & 1 \\
\hline
\end{tabular}

the eight cases, four flaps failed due to patient actions that cannot be attributed to the negative pressure dressing (-Table 4). One patient mobilized and dangled his lower extremity on postoperative day 3 prior to the scheduled dangle protocol. Another patient had a violent convulsive episode and avulsed the vascular pedicle on postoperative day 1 . A third patient rolled onto the flap in bed, leading to flap loss. Finally, one patient removed his splint and rested his limb directly on 
Table 3 Complications evaluated in this study with total number of flaps and percent of flaps calculated per complication

\begin{tabular}{|l|l|l|}
\hline Complication & $\begin{array}{l}\text { Total number } \\
\text { of flaps }\end{array}$ & $\begin{array}{l}\text { Percent of } \\
\text { flaps (\%) }\end{array}$ \\
\hline Flap failure & 8 & 8.4 \\
\hline STSG failure & 3 & 3.2 \\
\hline Hematoma & 1 & 1.1 \\
\hline Distal flap necrosis & 1 & 1.1 \\
\hline Inability to maintain seal & 1 & 1.1 \\
\hline Partial muscle necrosis & 1 & 1.1 \\
\hline Mild flap congestion & 1 & 1.1 \\
\hline
\end{tabular}

Abbreviation: STSG, split-thickness skin graft.

his free latissimus flap. The remaining four flaps that failed represent $4.1 \%$ of this series and potentially could be attributed to the negative pressure dressing ( - Table 4 ). One of these flap failures involved a latissimus dorsi muscle for foot reconstruction, which on return to the operating room had no identifiable thrombosis, and a diagnosis of vasospasm was made as the contributing cause to flap loss. A second foot reconstruction performed with a latissimus dorsi flap failed due to arterial thrombosis on postoperative day 1 . The third flap failure was due to an unknown cause and involved ankle reconstruction using a latissimus dorsi flap. This occurred after postoperative day 4 . The fourth flap failure involved a free gracilis flap to the leg that suffered venous congestion on postoperative day 0 , leading to irreversible ischemic damage and total flap loss. Although we can say that it is possible that the NPWT contributed to these four flap losses, we cannot definitively prove causation.

\section{Discussion}

Negative pressure therapy has revolutionized the management of wounds. As previously discussed, negative pressure therapy has provided an acceptable alternative to skin graft bolsters. Chim et al and $\mathrm{Bi}$ et al have also demonstrated that negative pressure therapy can be used reliably in free tissue transfer. $^{4,5}$ Indeed, we have additionally utilized NPWT around fasciocutaneous flaps in situations where trauma has precluded primary closure due to swelling. However, the limitations to the above mentioned studies were their small sample sizes. A systematic review by Yu et al identified 137 flaps in which NPWT was applied to free tissue transfers to facilitate flap take and reduce complications. They found a complication rate of $5.7 \%$ with these flaps, suggesting that NPWT may be beneficial to flap attachment and in particular assist with salvage of flaps that are infected or complicated by venous congestion. ${ }^{7}$ To date, our series with 97 patients over a 9-year period is the largest group of patients reported. The vast majority of wounds that were covered in our series involved in the lower extremity (83 of 97 flaps). Vascular compromise of lower extremity free flaps is cited anywhere from 15 to $20 \%$, with overall flap survival in this setting being approximately $95 \%{ }^{8-10}$ This correlates with a recent study by Xiong et al where 1,356 adult patients with 1,397 lower extremity free flap reconstructions from 30 publications were reviewed. The rate of total flap loss in their review was $6.0 \%{ }^{11}$ In another recent report, Cho et al experienced an $8 \%$ free flap loss rate in 518 lower extremity reconstructions. ${ }^{12}$ We had an overall flap failure rate of $8.4 \%$. Excluding the four flaps in our series that failed as a direct result of patient actions, our failure rate was $4.1 \%$, all in the lower extremity. These four flaps had other signs of flap compromise when the negative pressure wound dressings were removed. No clear or definitive statements can be made about whether the NPWT contributed to the failures, but it also cannot be ruled out. These failure rates are consistent with the accepted failure rates for lower extremity free flap reconstruction. This demonstrates that our flap failure rate while using negative pressure therapy is consistent with that of free flaps not using negative pressure dressings. Similarly, an analysis of free flap failure using National Surgical Quality Improvement Program (NSQIP) database by Kwok and Agarwal evaluated free flaps from multiple sites (head and neck, extremities, trunk, and breast) and by type (muscle, fascial, skin, bone, and bowel) from 2005 to 2010 and found that the overall flap failure rate was $5.1 \%$ with 1,187 flaps used in data analysis. Overall, the failure rate for muscle flaps from the NSQIP data analyzed

Table 4 Detailed descriptions of eight flap failures identified in this 9-year study

\begin{tabular}{|l|l|l|l|l|l|}
\hline Patient & Age & Date of flap & Wound & Free flap & Cause of failure \\
\hline 30 & 75 & January 3, 2012 & Ankle & Gracilis & Mobilized/dangled foot on postoperative day 3 \\
\hline 34 & 45 & May 28, 2012 & Foot & Latissimus dorsi & Violent episode, avulsed pedicle postoperative day 1 \\
\hline 40 & 49 & May 23, 2013 & Back & Vastus lateralis & Rolled onto flap \\
\hline 45 & 22 & January 21, 2014 & Foot & Latissimus dorsi & $\begin{array}{l}\text { Failure on postoperative day 1, possible vasospasm; } \\
\text { no thrombosis identified }\end{array}$ \\
\hline 61 & 32 & December 20, 2014 & Forearm & Latissimus dorsi & Removed splint, rested on flap \\
\hline 74 & 35 & October 16, 2015 & Foot & Latissimus dorsi & Arterial thrombosis postoperative day 1 \\
\hline 81 & 50 & June 6, 2016 & Ankle & Latissimus dorsi & Unknown cause, failure after postoperative day 4 \\
\hline 83 & 36 & February 16, 2018 & Leg & Gracilis & $\begin{array}{l}\text { Venous congestion on postoperative day 0, } \\
\text { ischemic damage irreversible, total flap loss }\end{array}$ \\
\hline
\end{tabular}


was 4.7\%. However, Kwok and Agarwal cited several other studies in which the failure rates ranged from 0 to $8.4 \%{ }^{13}$ Accordingly, the overall flap loss rate for all muscle flaps from all sites in our series was $8.4 \%$, which demonstrates that the overall flap loss rate with negative pressure dressings utilized is similar to that of muscle flaps where negative pressure was not used.

As discussed earlier, Blackburn et al in 1998 reported 95\% skin graft take when using negative pressure therapy as a bolster for skin grafts. ${ }^{3}$ In another case series by Hanasono et al, they demonstrated that negative pressure therapy applied to skin grafts over microvascular free muscle flaps had between 98 and $100 \%$ skin graft take. ${ }^{14}$ We found that in our series, splitthickness skin graft failure occurred in $3.2 \%$ of flaps which is comparable to the Blackburn and Hanasono findings. The negative pressure dressing helps to prevent shearing forces and removes degradation byproducts while at the same time encouraging neovascularization and controlling edema with minimal skin graft loss. Unlike the Hanasono series, we were able to successfully and easily monitor our free flaps by creating windows in the foam sponge. Likewise, the rates of hematoma, distal flap necrosis, inability to maintain negative pressure seal, partial muscle necrosis, and mild flap congestion were all $1.1 \%$ in our study, which is relatively low demonstrating that negative pressure dressing therapy does not significantly contribute to these complications.

\section{Conclusion}

Given the low complication rates with the use of negative pressure dressings over free muscle flaps in our series and that these rates are comparable to studies in which negative pressure therapy was not used, we believe that negative pressure therapy is safe and effective to use with free muscle flaps undergoing immediate split-thickness skin grafting. The dressing protects muscle flaps and skin grafts postoperatively in a less bulky manner than conventional bolsters while at the same time controlling edema and fostering neovascularization. We have also demonstrated that the technique we have employed allows for easy postoperative assessment via Doppler monitoring and visual inspection. This is the largest series to date over an extended period of time and shows that negative pressure therapy can be a useful adjunct in the postoperative manage- ment of free muscle flaps with immediate split-thickness skin grafting.

Conflict of Interest

None declared.

\section{References}

1 Lalezari S, Lee CJ, Borovikova AA, et al. Deconstructing negative pressure wound therapy. Int Wound J 2017;14(04):649-657

2 Nakayama Y, Iino T, Soeda S. A new method for the dressing of free skin grafts. Plast Reconstr Surg 1990;86(06):1216-1219

3 Blackburn JH II, Boemi L, Hall WW, et al. Negative-pressure dressings as a bolster for skin grafts. Ann Surg 1998;40(05):453-457

4 Chim H, Zoghbi Y, Nugent AG, Kassira W, Askari M, Salgado CJ. Immediate application of vacuum assisted closure dressing over free muscle flaps in the lower extremity does not compromise flap survival and results in decreased flap thickness. Arch Plast Surg 2018;45(01):45-50

$5 \mathrm{Bi} \mathrm{H}$, Khan M, Li J, Pestana IA. Use of incisional negative pressure wound therapy in skin-containing free tissue transfer. J Reconstr Microsurg 2018;34(03):200-205

6 Eisenhardt SU, Momeni A, Iblher N, et al. The use of the vacuumassisted closure in microsurgical reconstruction revisited: application in the reconstruction of the posttraumatic lower extremity. J Reconstr Microsurg 2010;26(09):615-622

7 Yu P, Yu N, Yang X, Jin X, Lu H, Qi Z. Clinical efficacy and safety of negative-pressure wound therapy on flaps: a systematic review. J Reconstr Microsurg 2017;33(05):358-366

8 Gorman PW, Barnes CL, Fischer TJ, McAndrew MP, Moore MM. Soft-tissue reconstruction in severe lower extremity trauma. A review. Clin Orthop Relat Res 1989;243(243):57-64

9 Melissinos EG, Parks DH. Post-trauma reconstruction with free tissue transfer-analysis of 442 consecutive cases. J Trauma 1989; 29(08):1095-1102, discussion 1102-1103

10 Rainer C, Schwabegger AH, Bauer T, et al. Free flap reconstruction of the foot. Ann Plast Surg 1999;42(06):595-606, discussion 606-607

11 Xiong L, Gazyakan E, Kremer T, et al. Free flaps for reconstruction of soft tissue defects in lower extremity: A meta-analysis on microsurgical outcome and safety. Microsurgery 2016;36(06):511-524

12 Cho EH, Shammas RL, Carney MJ, et al. Muscle versus fasciocutaneous free flaps in lower extremity traumatic reconstruction: a multicenter outcomes analysis. Plast Reconstr Surg 2018;141 (01):191-199

13 Kwok AC, Agarwal JP. An analysis of free flap failure using the ACS NSQIP database. Does flap site and flap type matter? Microsurgery 2017;37(06):531-538

14 Hanasono MM, Skoracki RJ. Securing skin grafts to microvascular free flaps using the vacuum-assisted closure (VAC) device. Ann Plast Surg 2007;58(05):573-576 